\section{FRI0120 SERUM CALPROTECTIN IS NOT PREDICTIVE FOR SUCCESSFUL DOSE REDUCTION OR DISCONTINUATION OF TNF INHIBITORS IN RA PATIENTS WITH LOW DISEASE ACTIVITY}

N. Den Broeder ${ }^{1}$, L. Tweehuysen ${ }^{1}$, T. Vog| ${ }^{2}$, N. van Herwaarden ${ }^{1}$, F.H.J. van den Hoogen ${ }^{1,3}$, R.M. Thurlings ${ }^{3}$, A.A. den Broeder ${ }^{1} .{ }^{1}$ Rheumatology, Sint Maartenskliniek, Nijmegen, Netherlands; ' 2 University of Münster, Münster, Germany; ${ }^{3}$ Rheumatology, Radboudumc, Nijmegen, Netherlands

Background: Dose reduction and discontinuation of TNF inhibitors (TNFi) have been shown feasible in a large proportion of RA patients with low disease activity. ${ }^{1}$ However, to date, no predictors for successful dose reduction or discontinuation have been identified. ${ }^{2}$ Calprotectin (a heterodimer of S100A8/S100A9) might be a promising biomarker in this context, as preliminary data showed this pro-inflammatory marker to be associated with disease activity and radiographic progression in RA and to be predictive for response in RA patients starting treatment with a TNFi. 3,4

Objectives: To investigate the predictive value of baseline serum calprotectin for successful TNFi dose reduction or discontinuation in RA patients with low disease activity.

Methods: Data was derived from the intervention arm of the DRESS (Dose REduction Strategies of Subcutaneous TNFi) study, which showed non-inferiority of a dose reduction strategy of adalimumab or etanercept compared to usual care. ${ }^{1}$ TNFi dose interval was reduced stepwise every 3 months until flare (DAS28-CRP increase $>1.2$ or $>0.6$ if current DAS28-CRP $\geq 3.2$ ) or discontinuation. Patients were classified at 18 months as being successfully dose reduced, discontinued or not able to reduce the TNFi dose. At baseline, quantification of calprotectin was carried out on serum samples using ELISA. To assess the predictive value, calprotectin levels were compared between each group and receiver-operatorcharacteristic (ROC) curves were created. In addition, calprotectin was correlated cross-sectionally with several clinical markers for disease activity.

Results: Calprotectin levels were available for 102 of 121 patients randomised to the intervention group; $61 \%$ were women, $63 \%$ received etanercept and $37 \%$ received adalimumab. Overall, $46 \%$ of patients successfully reduced their TNFi dose, $19 \%$ of patients successfully discontinued their TNFi and $35 \%$ of patients could not reduce their TNFi dose. In these groups, median calprotectin levels were $599 \mathrm{ng} / \mathrm{ml}$ (p25-p75: 473-965), $629 \mathrm{ng} / \mathrm{mL}$ (p25-p75: 453-896) and 624 $\mathrm{ng} / \mathrm{mL}$ (p25-p75: 514-931) $(\mathrm{p}=0.801)$ (Figure 1). The area under the ROC-curve was 0.52 (95\% Cl: $0.40-0.63)$ for predicting successful TNFi dose reduction, 0.53 (95\% Cl: $0.38-0.67)$ for successful TNFi discontinuation and $0.54(95 \% \mathrm{Cl}$ : $0.42-0.66$ ) for no dose reduction possible. Calprotectin levels were significantly but weakly correlated with C-reactive protein (CRP) levels with a Spearman $\rho$ of $0.21(p=0.03)$. No significant correlation was found between calprotectin and age, gender, DAS28-CRP, rheumatoid factor or ACPA positivity.

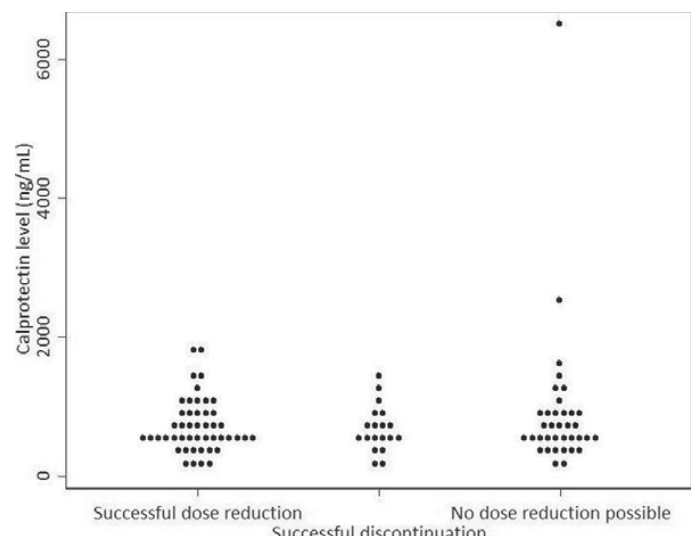

Conclusions: Serum calprotectin is not predictive for successful TNFi dose reduction or discontinuation in the context of RA patients with low disease activity, and calprotectin was only weakly correlated to CRP levels. These results might be caused by the lack of variability in calprotectin levels at baseline as all patients were in low disease activity state.

References:

[1] van Herwaarden et al. BMJ 2015;350:h1389.

[2] Tweehuysen et al. Arthritis Rheumatol 2016.

[3] Berner Hammer et al. Ann Rheum Dis 2010;69(1):150-4.

[4] Choi et al. Ann Rheum Dis 2015;74(3):499-505.

Disclosure of Interest: N. Den Broeder: None declared, L. Tweehuysen: None declared, T. Vogl: None declared, N. van Herwaarden: None declared, F. van den Hoogen Consultant for: Biogen, Celltrion, Janssen, Mundipharma and Sandoz, R. Thurlings: None declared, A. den Broeder Consultant for: AMGEN

DOI: 10.1136/annrheumdis-2017-eular.1729

\section{FRI0121 REMISSION AT 6 MONTHS AND IDENTIFICATION OF FUTURE GOOD RADIOGRAPHIC AND PHYSICAL OUTCOME IN EARLY RHEUMATOID ARTHRITIS}

N.P. Sundlisater ${ }^{1}$, A.-B. Aga ${ }^{1}$, I.C. Olsen ${ }^{1}$, H.B. Hammer ${ }^{1}$, T. Uhlig ${ }^{1}$, D. van der Heijde $^{2}$, T.K. Kvien ${ }^{1}$, S. Lillegraven ${ }^{1}$, E.A. Haavardsholm ${ }^{1}$ on behalf of the ARCTIC study group. ${ }^{1}$ Diakonhjemmet Hospital, Oslo, Oslo, Norway; ${ }^{2}$ Leiden University Medical Center, Leiden, Netherlands

Background: Treat-to-target strategies with achievement of clinical remission within 6 months minimize radiographic progression and physical impairment in RA.(1) The preferred target, ACR/EULAR Boolean remission criteria, was developed to optimize a combination of radiographic and functional outcome.(2) However, clinical remission does not exclude subclinical inflammation, associated with progression of radiographic damage.(3)

Objectives: Our aim was to assess the association between definitions of remission at six months, including absence of ultrasound inflammation, and future absence of joint damage and physical disability.

Methods: DMARD-naïve RA patients with $<2$ years since first swollen joint and indication for DMARD treatment were included in the treat-to-target ARCTIC trial.(4) Patients followed with ultrasound (treatment target DAS $<1.6+$ SJC44=0 + no PD signal in 32 joints) were analyzed. We assessed remission at 6 months according to the following criteria: DAS-ESR, DAS28-ESR, SDAI, CDAI, ACR/EULAR Boolean remission (based on 44 joints), no swollen joints (SJC44), no ultrasound PD signal in any joint (0-96), and minimal greyscale (GS) score (GS $\leq 2,0-96)$. Radiographs were scored by two readers using van der Heijde-Sharp score, with no radiographic progression defined as $<1$ unit change between 12-24 months based on the average score of the readers. We defined good outcome as a combination of no radiographic progression and physical function (PROMIS) $\geq$ median of the general population.(2) Odds ratios of reaching both outcomes were calculated according to various remission states at 6 months.

Results: Of the 103 patients included, 74\% were female, mean [SD] age 51.4 [12.9], disease duration 6.7 [5.3] months, DAS 3.5 [1.1] and PROMIS 39 [8]. Median [25th, 75th percentile] radiographic progression 12-24 months was 0.49 $[0.0,1.03]$, and $71 \%$ had no progression. DAS remission was achieved by $59 \%$ at the 6 -month visit, while $42 \%$ were in ACR/EULAR Boolean remission. Patients in ACR/EULAR Boolean remission had significantly higher odds ratio of no radiographic progression from 12-24 month than patients not in Boolean remission, as had patients with no PD signal or minimal GS findings (fig). Being in remission at six months according to each composite score separately or ACR/EULAR Boolean remission (OR 7.52, Cl $3.06-18.49$ ) predicted a good outcome, while criteria based on ultrasound or no swollen joints did not (fig). No statistically significant association was found between remission according to composite scores and radiographic progression (Fig).

Figure: $O R$ of no radiographic progression at 12-24 months compared to $O R$ of a good outcome (no radiographic progression + physical function $\geq$ median in the general population) at 12-24 months. P-values $\leq 0.05$ marked with an asterix.

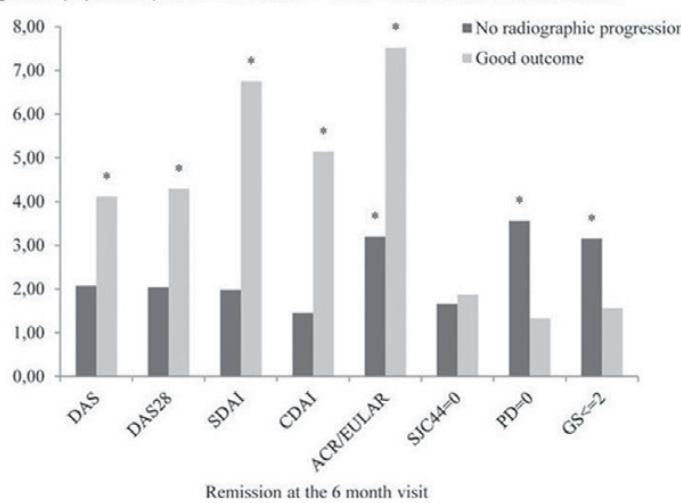

Conclusions: Our data show that being in ACR/EULAR Boolean remission after six months of treat-to-target therapy increases both the odds of no radiographic progression and good physical function, supporting current recommendations stating that ACR/EULAR remission should be the preferred treatment target in early RA.(1) Additionally, our analyses support that absence of ultrasound inflammation is associated with no future radiographic progression.

References:

[1] Smolen JS, et al. ARD. 2016;75:3-15.

[2] Felson DT, et al. ARD. 2011;70:404-13.

[3] D'Agostino M, et al. ARD. 2016;75:1902-1908

[4] Haavardsholm E, et al. BMJ. 2016;354:i4205.

Disclosure of Interest: N. Sundlisater: None declared, A.-B. Aga: None declared, I. Olsen: None declared, H. Hammer Consultant for: AbbVie, Pfizer, BMS, Roche, UCB, T. Uhlig: None declared, D. van der Heijde: None declared, T. Kvien: None declared, S. Lillegraven: None declared, E. Haavardsholm Grant/research support from: AbbVie, Pfizer, MSD, Roche, UCB

DOI: 10.1136/annrheumdis-2017-eular 2834 